Check for updates

Cite this: RSC Adv., 2018, 8, 24993

Received 28th February 2018

Accepted 29th May 2018

DOI: $10.1039 / \mathrm{c} 8 \mathrm{ra01788c}$

rsc.li/rsc-advances

\section{Covalently-functionalized graphene oxide via introduction of bifunctional phosphorus- containing molecules as an effective flame retardant for polystyrene}

\author{
Kang Dai, (DD *a Shuai Sun, ${ }^{a}$ Wenbin Xu, ${ }^{\text {*a }}$ Yuan Song, ${ }^{a}$ Zhenzhen Deng $^{a}$ \\ and Xiaodong Qian ${ }^{\text {b }}$
}

Covalently-functionalized graphene oxide (FGO) was successfully prepared by grafting a novel phosphorus-containing flame retardant, [2-((6-oxidodibenzo[c,e][1,2]oxaphosphinin-6-yl)methoxy) acryloxyethylchlorophosphate, PACP], to graphene oxide (GO). The resulting FGO demonstrated hydrophobicity and stability in polar solvents such as $N, N$-dimethylformamide (DMF). The reactive vinyl groups of PACP attached to FGO further copolymerized with styrene to produce polystyrene-FGO (PSFGO) nanocomposites. PS-FGO samples showed obviously improved fire-resistance, thermal behavior and glass transition temperature in comparison with those of neat PS and PS-GO samples, due to the good dispersion of FGO in PS as well as the strong interfacial bonds between FGO and the matrix. In addition, thermogravimetry-Fourier transform infrared (TG-FTIR) results indicated that the evolution of volatile products from PS decomposition was significantly inhibited by the introduction of FGO. Furthermore, scanning electron microscopy (SEM) and FTIR and Raman spectroscopy were employed to investigate the char residue, elaborating the flame-retardance mechanism.

\section{Introduction}

Nowadays, nanotechnology is proved to be an efficient way to improve the fire safety resistance performance of materials, because only adding a small amount of nanofillers can significantly improve the thermal stability and combustion performance of materials. ${ }^{1-3}$ Recently, graphene has attracted wide public interest, due to its high strength, conductivity and specific surface area, attractive for improving the mechanical, thermal, and gas barrier properties of polymer materials..$^{4,5}$ In recent years, graphene and its derivatives as flame retardants have been extensively applied in various polymers to improve their fire safety. The single addition of graphene, however, is prone to agglomerate, which is attributed to the strong van der Waals attractions among graphene sheets. ${ }^{6,7}$ This drawback will give rise to the nonuniform dispersion of graphene and unfavorable graphene-matrix interfacial adhesion, which will further weaken the property enhancement of graphene-based nanocomposites and limit the utilization of graphene to a great extent. As a widely employed strategy to solve this issue, surface functionalization of graphene has drawn the attention of many researchers over the years.

${ }^{a}$ School of Environmental Science and Engineering, Guangdong University of Technology, Guangzhou, Guangdong, 510006, P. R. China. E-mail: xuwenbin@gdut. edu.cn; daikang@mail.ustc.edu.cn; Fax: +86 20 39322296; Tel: +86 2039322296 ${ }^{b}$ Chinese People's Armed Police Force Academy, Langfang, Heibei, 065000, P. R. China
Generally, surface functionalization includes non-covalent and covalent functionalization. ${ }^{\mathbf{2}}$ Non-covalent functionalization weakens the van der Waals force between graphene sheets, thereby enhancing their dispersion in solvents and polymers. Nevertheless, non-covalent interactions including hydrophobic and electrostatic interactions, as well as $\pi-\pi$ stacking can cause a weak adhesion for the graphene-matrix interface, giving rise to a decrease in the interfacial load transfer. Moreover, the enhancement effect on the properties of composites is debilitated by the residual additive modifiers (e.g., surfactants). In comparison with that, covalent functionalization involves the introduction of tailored functional molecules to graphene or graphene-based materials via covalent linkages. The strongly attached chains can further chemically bond with the polymeric matrix, serving as connections for graphene and the matrix to form an integral part. The resulting nanocomposites mostly demonstrate conspicuous enhancement in properties. Consequently, covalent functionalization distinguishes from other approaches in improving the properties of materials as reported by researchers. Bao et al.9,10 prepared FGO via grafting hexachlorocyclo-, triphosphazene and hydroxyethyl acrylate (HEA) to GO. The produced nanocomposites with FGO demonstrate notable enhancement in both peak heat release rate (PHRR) and total heat release (THR) reduction as well as char formation increase. Yuan et al. ${ }^{11}$ reported the preparation of FGO/polypropylene (PP) nanocomposites. In a $2.0 \mathrm{wt} \%$ amount of addition, the FGO modified with melamine contributes a $29 \%$ 
decrease to PHRR as compared with that of pure PP, and the fire resistance of these nanocomposites is also improved obviously.

For DOPO and its derivates, these phosphorus-based flame retardants can exert flame retardant action in gas phase. ${ }^{\mathbf{1 2}}$ In condensed phase flame retardant action, phosphate species are capable of catalyzing acrylic derivates to form cohesive char layers for the protection of underlying polymer matrix. ${ }^{13-15}$ Consequently, combining both condensed and gas phases actions will be conductive to the fire-resistance performance of flame retardants.

Polystyrene (PS) with prominent thermal and chemical resistance, low density, good mechanical properties and convenience of processing and molding, has been widely applied in industrial fields. Whereas, the high flammability and subsequent severe dripping combined with the released heavy smoke and toxic gas during combustion greatly limit the further application of PS in some fields. Therefore, it is imperative to develop highperformance flame retardants to reduce the flammability of PS.

\section{Experimental}

\subsection{Materials}

GO was obtained using a modified Hummers' method, ${ }^{16,17}$ and graphite powder with an average particle size of 800 mesh and a purity of $>99 \%$ was supplied by Aladdin reagent Co. Ltd (Shanghai, China). Potassium permanganate $\left(\mathrm{KMnO}_{4}\right)$ was purchased from Kaixin chemical reagent Co. Ltd (Hengyang, China). Hydrochloric acid $(\mathrm{HCl})$, sulfuric acid $\left(\mathrm{H}_{2} \mathrm{SO}_{4}\right)$, barium chloride $\left(\mathrm{BaCl}_{2}\right)$ and anhydrous ethanol were purchased from Guangzhou chemical reagent factory. Sodium nitrate $\left(\mathrm{NaNO}_{3}\right)$ was supplied by Shanghai Taitan chemical Co. Ltd. Hydrogen peroxide $\left(\mathrm{H}_{2} \mathrm{O}_{2}, 30 \%\right.$ aq.) dimethylbenzene, $\mathrm{N}, \mathrm{N}$-dimethylformamide (DMF), triethylamine (TEA) and styrene were purchased from Damao Chemical Reagent Factory (Tianjin, China). 9,10Dihydro-9-oxa-10-phosphaphenanthrene-10-oxide (DOPO) was supplied by Shenzhen Jinlong Chemical Co. Ltd (Shenzhen, China). Paraformaldehyde (HCHO) and benzoyl peroxide (BPO) was supplied by Aladdin reagent Co. Ltd (Shanghai, China). Hydroxyethyl acrylate (HEA) and phosphorus oxychloride were provided by Yangzhou Weide Chemical Raw Material Co. Ltd (Shanghai, China). Styrene was purified before used.

\subsection{Synthesis of 6-(hydroxymethyl) dibenzo $[c, e][1,2]$ oxaphosphinine 6-oxide (DOPO-OH)}

Firstly, DOPO (43.234 g, $0.2 \mathrm{~mol}$ ) and dimethyl benzene (100 ml) were added into a $500 \mathrm{ml}$ three-necked glass flask which was equipped with a mechanical stirrer, a reflux condenser and a nitrogen inlet. The mixture was stirred and heated slowly to $90{ }^{\circ} \mathrm{C}$ for $1 \mathrm{~h}$ under nitrogen atmosphere until DOPO was dissolved completely in dimethyl benzene. Next, paraformaldehyde (6.006 g, $0.2 \mathrm{~mol}$ ) was added into the mixture. The reaction mixture was heated to $140{ }^{\circ} \mathrm{C}$ and kept at the refluxing temperature for another 6 h. Finally, the precipitate was filtered and washed with dimethyl benzene after cooling to room temperature, yielding a white powder. The powder was dried under a vacuum at $70{ }^{\circ} \mathrm{C}$ for $12 \mathrm{~h}$.

The synthetic procedures for DOPO-OH are illustrated in Scheme 1.

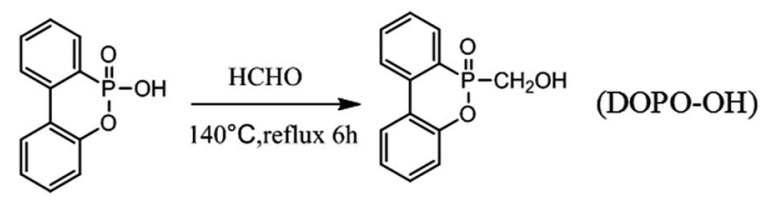

Scheme 1 Synthesis route of DOPO-OH.

\subsection{Synthesis of FGO}

In an ice bath machine $\mathrm{POCl}_{3}(9.200 \mathrm{~g}, 0.06 \mathrm{~mol})$ and DMF (70 $\mathrm{ml}$ ) were firstly fed into a $500 \mathrm{ml}$ three-neck flask equipped with a dropping funnel, a nitrogen inlet and a mechanical stirrer. The mixture was stirred vigorously and cooled to $-5{ }^{\circ} \mathrm{C}$ under dry $\mathrm{N}_{2}$. Then a mixture of TEA $(20.238 \mathrm{~g}, 0.20 \mathrm{~mol})$ and DMF (20 $\mathrm{ml}$ ) was added. After $20 \mathrm{~min}$, DOPO-OH (14.776 g, $0.06 \mathrm{~mol}$ ) dissolved in DMF $(60 \mathrm{ml})$ were then added dropwise for $2 \mathrm{~h}$ and maintained at this temperature for an additional $4 \mathrm{~h}$. After that, another mixture of HEA (6.967 g, $0.06 \mathrm{~mol}$ ) and DMF (40 ml) was slowly added, and the resulting mixture was maintained stirring for additional $4 \mathrm{~h}$ to obtain PACP. Then the mixture was heated to room temperature. GO $(1.0 \mathrm{~g})$ was added and kept stirring for another $12 \mathrm{~h}$. Finally, the mixture was filtered and washed thoroughly with deionized water. The resultant product was dried under a vacuum at $35^{\circ} \mathrm{C}$ for $24 \mathrm{~h}$, yielding a black powder (FGO, illustrated in Scheme 2a).

\subsection{Preparation of PS-GO and PS-FGO nanocomposites}

In different ratios, GO/FGO was mixed with styrene to prepare PS-GO and PS-FGO nanocomposites, and BPO was used as an initiator at a concentration of $2 \mathrm{wt} \%$. Each mixture was stirred vigorously with ultrasonication for $2 \mathrm{~h}$. Then the mixture was heated to $90{ }^{\circ} \mathrm{C}$ and stirred until a critical viscosity. Subsequently, all samples were cast into polytetrafluoroethylene molds, cured at $90{ }^{\circ} \mathrm{C}$ for $8 \mathrm{~h}$ and post cured at $120{ }^{\circ} \mathrm{C}$ for $4 \mathrm{~h}$. According to the amount of GO/FGO in nanocomposites, the cured samples are referred to hereafter as and PS-GO1.0, PSFGO1.0, PS-FGO2.0 and PS-FGO3.0. The schematic reaction route between FGO and styrene is presented in Scheme $2 \mathrm{~b}$.

(a)

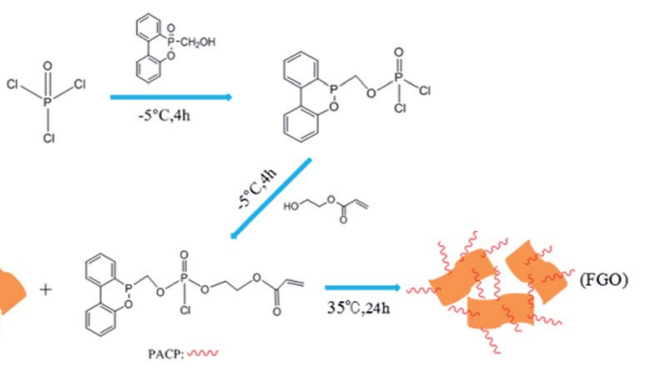

(b)

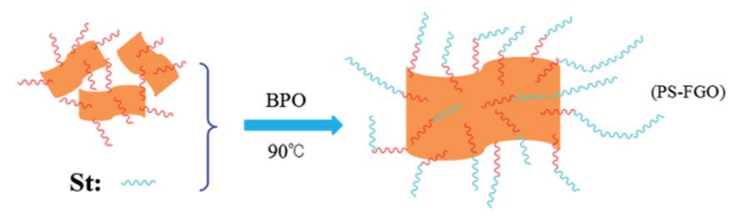

Scheme 2 Synthesis routes of (a) FGO and (b) PS-FGO nanocomposites. 


\subsection{Characterization}

Fourier transform infrared (FTIR) spectra were recorded with a Nicolet 6700 spectrometer (Nicolet Instrument Company, USA). For solid samples, KBr discs were prepared. The ${ }^{1} \mathrm{H}$ NMR spectrum was recorded using a Bruker AVANCE III $400 \mathrm{MHz}$, and the spectrum was referenced by solvent shifts $\left(\right.$ DMSO- $_{6}=$ $2.50 \mathrm{ppm}$ ). The morphologies of samples were examined by a SEM solver p47 pro using charge contrast imaging mode. Transmission electron microscopy (TEM) analysis was performed on a JEM-2100 transmission electron microscope at 200 $\mathrm{kV}$. X-ray diffraction (XRD) experiments were conducted on a D/ MAX-IIIC X-ray diffractometer with $\mathrm{Cu} \mathrm{K} \alpha$ radiation. Thermal gravimetric analysis (TGA) was conducted on a PE TGA-7 calorimeter from room temperature to $700{ }^{\circ} \mathrm{C}$ at a linear heating rate of $20{ }^{\circ} \mathrm{C} \mathrm{min}^{-1}$ under air or nitrogen atmosphere. Differential scanning calorimetry (DSC) was performed on a DSC Q2000 instrument (TA Ltd., USA) at a heating rate of $10{ }^{\circ} \mathrm{C} \min ^{-1}$ under nitrogen atmosphere. Micro combustion calorimeter (MCC) test was carried out in a MCC-2 (Govmark Inc., New York, USA). According to ASTM D 7309, 5-7 mg of powdery samples were heated to $700{ }^{\circ} \mathrm{C}$ at a heating rate of $1{ }^{\circ} \mathrm{C}$

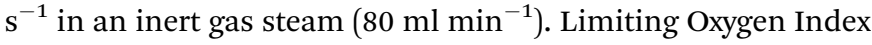
(LOI) was measured using a HC-2C limiting Oxygen Index Analyzer instrument on test bars of size 100 it $6.5 \times 3.0 \mathrm{~mm}^{3}$, according to the ASTM standard D2863-77. Raman spectroscopy measurements were conducted at room temperature using a Lab RAMHR 800UV Laser Raman spectrometer with a laser wavelength of $633 \mathrm{~nm}$, and the Gaussian peak type in the Peak Fitting Module of Origin 8.0 software was employed in curve fitting to determine spectral parameters.

\section{Results and discussion}

\subsection{Structural and morphological characterization of DOPO-OH, GO, FGO and PS-GO/FGO nanocomposites}

The structural characterization of DOPO-OH on ${ }^{1} \mathrm{H}$ NMR spectrum is shown in Fig. 1. The peak at $3.35 \mathrm{ppm}$ is assigned to the proton of $-\mathrm{OH}$, and the chemical shifts $\delta=3.90-4.40 \mathrm{ppm}$ are attributed to the protons of $-\mathrm{CH}_{2}-$. Moreover, the peaks at 7.188.41 ppm belong to the $8 \mathrm{H}$ atoms of aromatic rings. ${ }^{18,19}$ All of these ${ }^{1} \mathrm{H}$ NMR results conform to the expected structure of DOPO-OH.

The chemical structures of GO and FGO were characterized by XRD. As shown in Fig. 2, for GO, in addition to a weak (100)

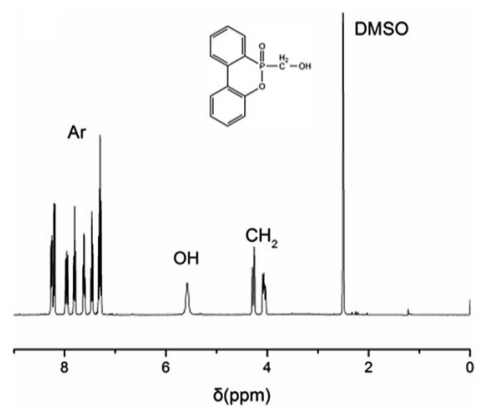

Fig. $1{ }^{1} \mathrm{H}$ NMR spectrum of DOPO-OH.

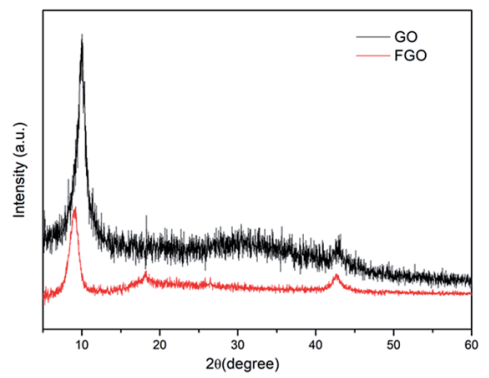

Fig. 2 XRD patterns of $\mathrm{GO}$ and FGO.

reflection peak at $42^{\circ}$, another sharp diffraction peak at scattering angle $2 \theta=10.1^{\circ}$ is observed, which corresponds to the (002) reflection of GO.$^{20}$ After functionalization, the intensity of the (002) peak in FGO significantly reduces, suggesting a decrease in the ordered arrangement of GO. Meanwhile, the (002) characteristic peak shifts to $9.0^{\circ}$, indicative of an enlarged interlayer spacing. This is accounted for by the intercalation of PACP molecules, which react with the hydroxyl groups and covalently bond on GO surface. Moreover, variations of the functionalized groups on GO and FGO are further corroborated by the FTIR spectroscopy measurements as shown in Fig. 3. Some typical absorption peaks characteristic of oxygencontaining groups can be clearly seen for GO: the stretching vibration of $-\mathrm{OH}\left(3390 \mathrm{~cm}^{-1}\right)$, the stretching vibration of $\mathrm{C}=\mathrm{O}$ $\left(1730 \mathrm{~cm}^{-1}\right), \mathrm{C}=\mathrm{C}$ or $\mathrm{H}_{2} \mathrm{O}$ vibration $\left(1623 \mathrm{~cm}^{-1}\right)$, the stretching vibration of $\mathrm{C}-\mathrm{O}-\mathrm{C}\left(1227 \mathrm{~cm}^{-1}\right)$ and $\mathrm{C}-\mathrm{OH}$ vibration $\left(1059 \mathrm{~cm}^{-1}\right) .{ }^{10}$ In contrast, the appearance of some new peaks can been found in the spectrum of FGO. In addition to the vibration of $\mathrm{C}-\mathrm{H}\left(2940-2980 \mathrm{~cm}^{-1}\right)$, the peaks at 1443, 1183 and $1036 \mathrm{~cm}^{-1}$ correspond to the stretching of $\mathrm{P}-\mathrm{C}, \mathrm{P}=\mathrm{O}$ and $\mathrm{P}-\mathrm{O}-$ $\mathrm{C}$, respectively. ${ }^{19,21}$ The emergence of these new characteristic peaks confirms the structure of FGO as shown in Scheme 2a.

The surface morphologies of GO and FGO were analyzed by TEM. The TEM image of GO (Fig. 4a) exhibits a typical transparent and wrinkled structure, revealing the presence of large single GO sheets. Different from the surface morphology of GO, after chemical functionalization the surface of FGO is found with some black shadows (Fig. 4b), and the typical layer structure, however, is not destroyed, which are in conformity with the FTIR results and further corroborate that these phosphorus-containing flame retardants were successfully grafted to the surface of GO. For analyzing the dispersion of nanosheets in polymer matrix, the fracture surfaces of samples were characterized by SEM. As shows

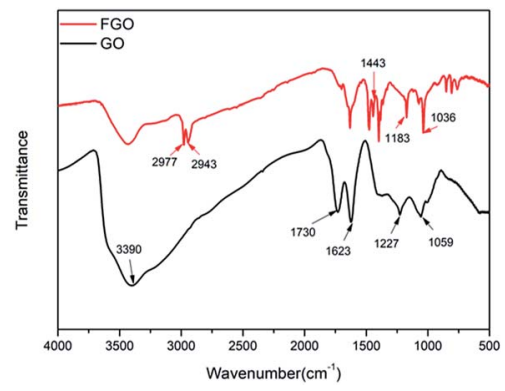

Fig. 3 FTIR spectra of GO and FGO. 


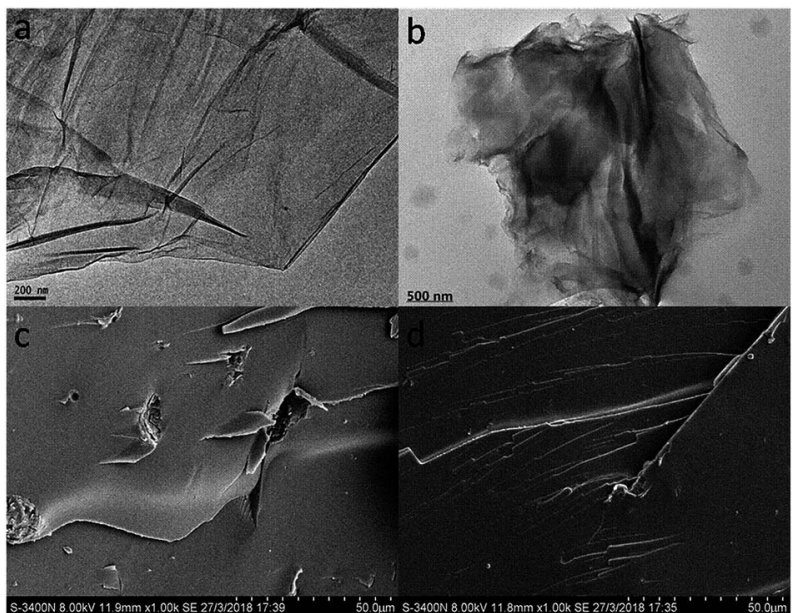

Fig. 4 TEM images of (a) GO and (b) FGO, and SEM images of the fractured surfaces of (c) PS-GO1.0 and (d) PS-FGO3.0.

in Fig. 4c, agglomerated GO nanosheets can be found in PS-GO1.0, due to the strong van der Waals attractions among GO nanosheets ${ }^{22}$ and the poor interfacial interaction between GO and PS molecular chains. In contrast, the PS-FGO3.0 fracture surface shows no pulling-out of nanosheets. Because of the covalently functionalized molecules on the surface of FGO, the repulsion forces among FGO nanosheets are enhanced, and FGO can be well dispersed in polymer matrix. Moreover, these functionalized flame retardant molecules covalently react with styrene monomers during polymerization. As a result, FGO nanosheets are integrated into PS molecular chains, leading to the disappearance of the interface between FGO and the matrix.

Because of the existence of numerous hydrophilic groups (e.g., hydroxyl and carboxyl groups), GO is readily exfoliated in aqueous media to form stable colloidal suspensions of thin sheets. ${ }^{23}$ It was observed that after ultrasonication and standing for 1 week, the GO can be stably dispersed in $\mathrm{H}_{2} \mathrm{O}$, as illustrated in Fig. 5 . In the case of FGO, in contrast, obvious precipitation can be seen in $\mathrm{H}_{2} \mathrm{O}$ for the hydrophobic nature, and it strongly tends to agglomerate. However, FGO can well suspend in polar solvents such as DMF, forming a deeply black solution. It can be explained by that the strongly polar moiety PACP is favorably dissolved in DMF, and the reaction between this phosphorus-containing molecule and GO improves the polarity of FGO. As a result, FGO shows much better dispersibility in DMF in contrast to that of GO.

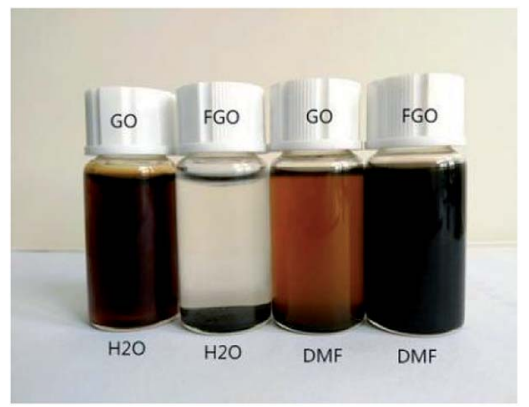

Fig. 5 Optical pictures of $\mathrm{GO}$ and FGO dispersed in $\mathrm{H}_{2} \mathrm{O}$ and DMF after ultrasonication for $5 \mathrm{~min}$ and waiting for 1 week.

\subsection{Thermal stability}

From the perspective of industrial application, the operating temperature range of a polymer material primarily depends on its $T_{\mathrm{g}}$. Specifically, a wide operating temperature range of a polymer material requires a high $T_{\mathrm{g}}$. The wrinkled surface of GO sheets (see Fig. 4a) with nanoscale surficial roughness facilitates the mechanical interlocking effect and constrains the mobility of polymer chains. ${ }^{24}$ Consequently, when combined with GO, PS-GO1.0 shows an increase of $T_{\mathrm{g}}$ value by $4{ }^{\circ} \mathrm{C}$ in comparison with the $82{ }^{\circ} \mathrm{C}$ of pure $\mathrm{PS},{ }^{25}$ as confirmed by DSC (Fig. 6). Interestingly, a higher $T_{\mathrm{g}}$ value in PS-FGO1.0 is observed, and the $T_{\mathrm{g}}$ value of PS-FGO nanocomposite increases from 90 to $102{ }^{\circ} \mathrm{C}$ when the amount of FGO reaches $3 \mathrm{wt} \%$, providing a $20^{\circ} \mathrm{C}$ increase for $T_{\mathrm{g}}$. The enhancement in $T_{\mathrm{g}}$ of PSFGO nanocomposites can be accounted for by two factors. In addition to the physical hindrance of PS chains movement by the nanosheets, PACP in FGO is covalently bonded with PS chains and plays the role of connection for GO and the polymer matrix. These combined effects contribute to a more significant restriction in the movement of PS chain segments. Therefore, much higher $T_{\mathrm{g}}$ values are shown in PS-FGO nanocomposites.

To understand the thermal behavior of PS after incorporation of GO/FGO, TGA was conducted to evaluate the thermal degradation process of all samples. The corresponding TG curves and detailed data are presented in Fig. 7 and Table 1, respectively. Under nitrogen condition, the TG curve of pure PS shows one-stage weight loss in the temperature range of 350$500{ }^{\circ} \mathrm{C}$. During this degradation, PS undergoes a chainscissoring process followed by depolymerization with the formation of styrene monomer, oligomers and some volatiles. ${ }^{26}$ Finally, a limited char residue is observed. For PS-GO1.0, due to the lower thermal stability of $\mathrm{GO},{ }^{27}$ the temperature at $10 \mathrm{wt} \%$ weight loss $\left(T_{0.1}\right)$ is somewhat lower than that of PS. Meanwhile, in comparison with the onset degradation temperatures of PSGO1.0, a decrease in $T_{0.1}$ of PS-FGO1.0 is observed, and this can be explained by the cleavage of the relatively weak $\mathrm{P}-\mathrm{O}-\mathrm{C}$ linkage in FGO, which can accelerate the degradation of the matrix. ${ }^{28}$ Different from the one-stage weight loss in PS, the twostage weight loss between 150 and $350{ }^{\circ} \mathrm{C}$ becomes increasingly evident with an increase in the FGO content of these samples. This indicates that the introduction of FGO into PS significantly alters the decomposition process. Moreover, the enhancement in char formation is worthy of note. At $700{ }^{\circ} \mathrm{C}$ only a $0.33 \mathrm{wt} \%$ char residue is seen for PS, however the char residue of PS-

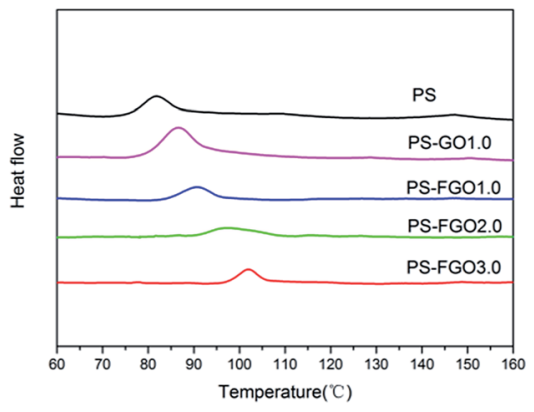

Fig. 6 DSC curves of PS and PS-GO/FGO nanocomposites. 

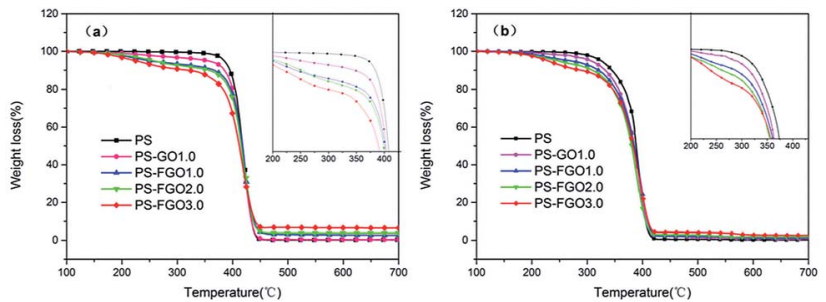

Fig. 7 TG curves of PS and PS-GO/FGO nanocomposites in (a) nitrogen and (b) air atmospheres.

FGO3.0 is as high as $6.50 \mathrm{wt} \%$. In addition, the $2.70 \mathrm{wt} \%$ char residue of PS-FGO1.0 is obviously higher than that of PS-GO1.0. This improvement is attributed to two factors: the GO nanosheets act as physical barriers to protect the PS matrix from thermal degradation; the flame retardant molecular chains on well dispersed FGO surface have a catalytic effect on char formation. The resulting high char residue with better thermal stability in condensed phase can retard the transfer of oxygen and flammable gaseous products, restrain thermal conductivity and endow higher decomposition temperatures to the matrix. ${ }^{29,30}$ Therefore, PS-FGO3.0 with the highest char residue exhibits the lowest maximum mass loss rate (MMLR). Specifically, the MMLR for PS-FGO3.0 is reduced by $28.5 \%$, relative to that of the neat PS. In air, the quantified temperatures [e.g., $T_{0.1}$, the temperature corresponding to the maximum weight loss $\left.\left(T_{\max }\right)\right]$ for all samples are somewhat lower than these under nitrogen, implying that these polymer materials degradation may be accelerated by oxygen, and the primary carbonaceous char further oxidizes after $430{ }^{\circ} \mathrm{C}$, as observed in Fig. 7-b. However, the thermo-oxidative stability of the samples with FGO at high temperatures is enhanced, as corroborated by the increased char residues at $700{ }^{\circ} \mathrm{C}$. This improvement in the inhibition of thermo-oxidative degradation of PS-FGO nanocomposites is attributed to the well dispersion of FGO as well as the strong interfacial interaction between FGO and PS, which hinders the thermal movement of polymer chains at high temperatures, promotes char formation and contributes to further enhancement in thermo-oxidative stability.

\subsection{Combustion properties}

The combustion performance of PS and PS nanocomposites was evaluated by LOI and MCC test. LOI is an important and representative parameter to evaluate the flammability of materials, ${ }^{31}$ and the LOI values of all samples are summarized in Table 2. The pure PS is high flammable with a low LOI value of
19.0. With the increase of FGO content, however, the LOI values of samples increase. The LOI value reaches 22.5 with $1 \mathrm{wt} \%$ amount of FGO and further increases to 25.0 for PS-FGO3.0, suggesting the flame retardancy of these nanocomposites is enhanced by the incorporation of FGO.

To further understand the combustion properties of all samples, MCC was used to evaluate their heat release. The heat release rate (HRR) curves of PS and PS nanocomposites and corresponding MCC data are presented in Fig. 8 and Table 2, respectively. It is evident that the total heat release (THR) of PSFGO nanocomposites decreases as the FGO amount rises. Meanwhile, it can be observed that the $23.4 \mathrm{~kJ} \mathrm{~g}^{-1}$ THR value of PSFGO1.0 is lower than that of PS-GO1.0. These results indicate that comparing to GO, FGO significantly facilitates the conversion degree of carbonization during combustion, resulting in more cohesive char instead of combustible volatiles, and fit well with the TGA experiments. Further, the reduced THR value suggests that less heat releases during combustion, which is beneficial to the control of potential fire hazard. For heat release capacity (HRC), as a good predictor of fire and flammability behavior, it is obtained by dividing the peak heat release rate by the heating rate and indicates the maximum capacity of a material to release combustion heat. ${ }^{32}$ While a high HRC value is observed for pure PS, introduction of FGO endows this polymer with a pronounced reduction in HRC. HRC value decreases from $984 \mathrm{~J} \mathrm{~g}^{-1} \mathrm{k}^{-1}$ (PS) to $599 \mathrm{~J} \mathrm{~g}^{-1}$ $\mathrm{k}^{-1}$ (PS-FGO3.0), with a reduction of $39.1 \%$. This improvement can be attributed to the reduced MMLR and the combustion heat of the decomposition products at that temperature. ${ }^{33}$ In the case of PS-FGO nanocomposites, as FGO can efficaciously catalyze char formation and hinder the thermal degradation of polymer matrix, less combustible gaseous products are formed with increasing FGO content. Thus reduced combustible volatiles coupled with the lowered MMLR contribute to the much better nonflammability of PS-FGO nanocomposites as compared to the neat PS. For PS-FGO nanocomposites with similar components, the difference in major decomposition products can be considered less than that in MMLRs. PS-FGO3.0 with the lowest MMLR, consequently, exhibits the best nonflammability.

The above results demonstrate that different from the mainly physical barrier effect of GO, well dispersed FGO can further react with the polymer molecule chains to improve the flame-retardant properties of PS-FGO nanocomposites.

\subsection{Flame-retardant mechanism analysis}

To understand the flame-retardant mechanism of PS-FGO nanocomposites, firstly, TG-FTIR spectroscopy is employed to

Table $1 T_{\mathrm{g}}$ and TGA data in nitrogen/air condition for PS and PS- GO/FGO nanocomposites

\begin{tabular}{|c|c|c|c|c|c|}
\hline Samples & $\begin{array}{l}T_{0.1}\left({ }^{\circ} \mathrm{C}\right) \\
\left(\mathrm{N}_{2} / \text { air }\right) \\
\end{array}$ & $\begin{array}{l}T_{\max }\left({ }^{\circ} \mathrm{C}\right) \\
\left(\mathrm{N}_{2} / \text { air }\right) \\
\end{array}$ & $T_{\mathrm{g}}\left({ }^{\circ} \mathrm{C}\right)$ & $\begin{array}{l}\operatorname{MMLR}\left(\% /{ }^{\circ} \mathrm{C}\right) \\
\left(\mathrm{N}_{2} / \text { air }\right)\end{array}$ & $\begin{array}{l}\text { Residues at } 700{ }^{\circ} \mathrm{C} \\
\text { (wt\%) }\left(\mathrm{N}_{2} / \text { air }\right)\end{array}$ \\
\hline Error & \pm 1 & \pm 1 & \pm 1 & \pm 0.01 & \pm 0.01 \\
\hline PS-GO1.0 & $384 / 332$ & $421 / 393$ & 86 & $2.56 / 1.96$ & $0.81 / 0.43$ \\
\hline PS-FGO1.0 & $364 / 320$ & $419 / 394$ & 90 & $2.47 / 1.86$ & $2.70 / 1.34$ \\
\hline PS-FGO2.0 & $354 / 309$ & $417 / 390$ & 97 & $2.23 / 1.80$ & $3.77 / 1.85$ \\
\hline
\end{tabular}


Table 2 Flame retardancy and MCC data of PS and PS-GO/FGO nanocomposites

\begin{tabular}{lllll}
\hline Sample & HRC $\left(\mathrm{J} \mathrm{g}^{-1} \mathrm{k}^{-1}\right)$ & THR $\left(\mathrm{kJ} \mathrm{g}^{-1}\right)$ & $T_{\text {pHRR }}\left({ }^{\circ} \mathrm{C}\right)$ & LOI $(\%)$ \\
\hline Error & \pm 1 & \pm 0.1 & \pm 1 & \pm 0.5 \\
PS & 984 & 26.7 & 441 & 19.0 \\
PS-GO1.0 & 799 & 24.2 & 441 & 21.0 \\
PS-FGO1.0 & 701 & 23.4 & 445 & 22.5 \\
PS-FGO2.0 & 631 & 22.7 & 438 & 24.0 \\
PS-FGO3.0 & 599 & 21.4 & 436 & 25.0
\end{tabular}

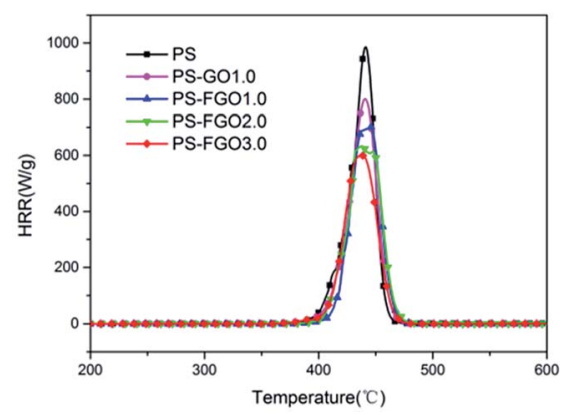

Fig. 8 HRR curves of PS and PS-GO/FGO nanocomposites.

analyze the evolved gaseous products of samples. Fig. 9 shows the $3 \mathrm{D}$ diagrams of the gaseous volatiles during combustion process of PS and PS-FGO3.0. It is evident that after incorporation of $\mathrm{FGO}$, the volatile products decrease significantly before $c a .400{ }^{\circ} \mathrm{C}$ (Fig. 9-b), and the volatile gas intensity reaches the maximum value at $c a .420{ }^{\circ} \mathrm{C}$, which is consistent with the MMLR temperature in TGA results. Characteristic FTIR signals of PS and PS-FGO3.0 pyrolysis products at MMLR were studied as presented in Fig. 10: $\mathrm{CO}_{2}\left(2360 \mathrm{~cm}^{-1}\right)$, compounds containing aromatic rings $\left(3073,1500,773\right.$, and $\left.698 \mathrm{~cm}^{-1}\right)$, and hydrocarbon groups $\left(2980-2900 \mathrm{~cm}^{-1}\right) \cdot{ }^{15,34} \mathrm{Jang}$ et al. ${ }^{35}$ reported that the mainly decomposed products of PS are styrene, dimer and trimer of phenyl alkenyl. Considering the DOPO moiety in FGO, it can exert flame retardant action in vapor phase. By comparing the spectra of both samples, although the characteristic peak of $\mathrm{P}-\mathrm{O}$ group cannot be clearly detected at high temperatures, because of overlap with the $\mathrm{C}-\mathrm{O}$ stretching band, it is observed that new adsorption band of $\mathrm{P}=\mathrm{O}\left(1290 \mathrm{~cm}^{-1}\right)^{12}$ appears in PS-FGO3.0, which is referred to $\mathrm{PO}_{2}$ in $\mathrm{H}_{3} \mathrm{PO}_{2}{ }^{18,36}$ This volatile phosphorus compound can act as an effective flame quencher to inhibit the gas-phase chain reactions in combustion, improving the flame retardancy of PS-FGO nanocomposites.
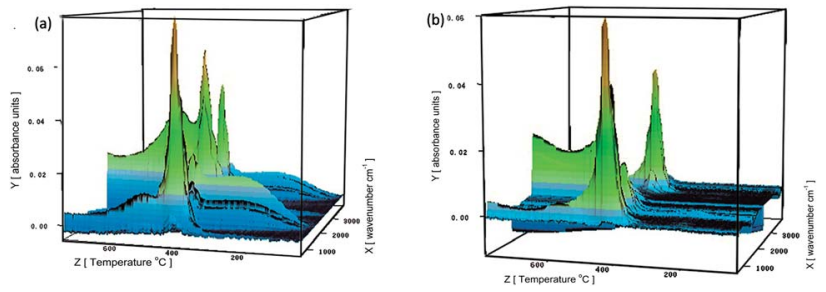

Fig. $93 \mathrm{D}$ diagrams of the gaseous volatiles during combustion process of (a) PS and (b) PS-FGO3.0.

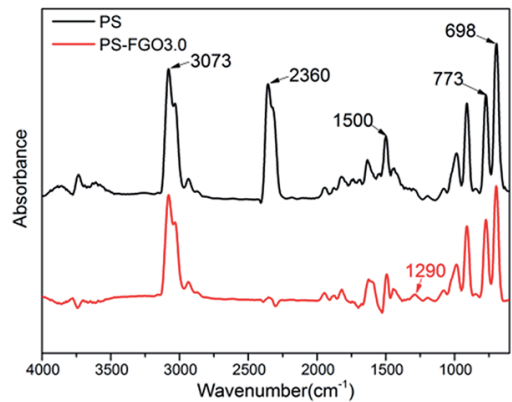

Fig. 10 FTIR spectra of pyrolysis products for PS and PS-FGO3.0 at MMLR.

In order to further investigate the variations of various volatiles, the absorbance-time of representative pyrolysis products (hydrocarbons, $\mathrm{CO}_{2}$, $\mathrm{CO}$ and aromatic compounds) for PS and PS-FGO3.0 were detected, and the absorbance values were normalized, as exhibited in Fig. 11. Clear indications of a remarkable reduction in the absorbance intensity of the combustible volatiles (hydrocarbons and aromatic compounds) are observed in PS-FGO3.0, as compared to these in PS. This improvement is attributed to the barrier effect of well dispersed FGO sheets and promoted char residues. As less combustible volatiles are evolved, HRR will be reduced, and MMLR can also be lowered. These results are in accordance with both TGA and MCC data. Moreover, the absorbance intensity of these smoke and toxic gases $\left(\mathrm{CO}_{2}\right.$ and $\left.\mathrm{CO}\right)$ in PS-FGO3.0 are much lower than these in PS, indicative of the prominent adsorption and barrier effects of FGO nanosheets as well as their strong interfacial bonds with matrix. Therefore, these much decreased heat, smoke and toxic gases in PS-FGO3.0 give evidence that incorporation of FGO can effectively reduce the fire hazard of PS-FGO nanocomposites.

Both char yield and char morphology are crucial to the flame retardant action of polymers in condensed phase. As the former TGA results show, increased char residues are seen for PS-FGO nanocomposites. To gain further insight into the char morphology of PS nanocomposites, corresponding char residues were prepared as reported previously and investigated by SEM. ${ }^{37}$ Fig. 12 shows SEM images of the micromorphologies of
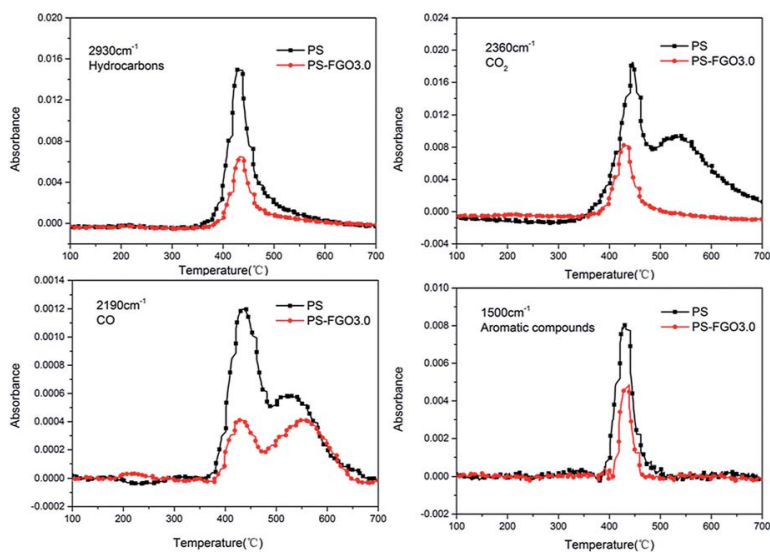

Fig. 11 Intensity of characteristic peaks of pyrolysis products for PS and PS-FGO3.0. 


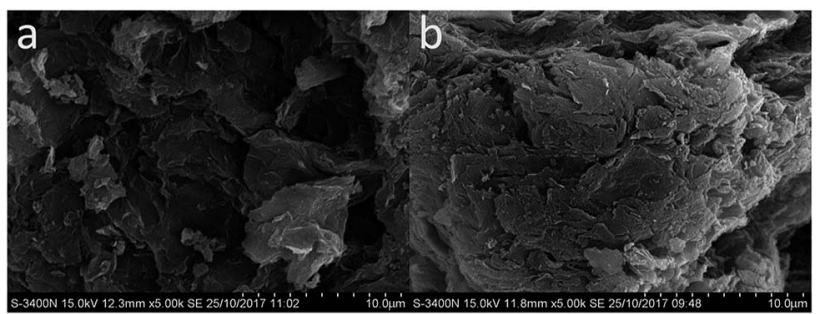

Fig. 12 SEM images of char residues: (a) PS-GO1.0 and (b) PS-FGO3.0.

PS-GO1.0 and PS-FGO3.0 char residues. As illustrated in Fig. 12a, the char residue of PS-GO1.0 appears to contain numerous pits and pores with a loose structure. PS-FGO3.0 char residue, in contrast, is much improved and demonstrates a compact and smooth surface (Fig. 12b), suggesting that FGO can catalyze the matrix to form cohesive char. Further, the microstructures of these char residues was investigated by Raman spectroscopy. As illustrated in Fig. 13, the spectra of both samples exhibit two highly overlapping diffusion bands, which are typical of graphitic phases. $^{38}$ The former, at ca. $1593 \mathrm{~cm}^{-1}$ (G band), represents the first order scattering $\mathrm{E}_{2 \mathrm{~g}}$ phonon of a $\mathrm{sp}^{2}$ hybrid carbon atom; in contrast, the latter, at ca.1323 $\mathrm{cm}^{-1}$ (D band), accounts for the oscillation of disordered carbon or glassy carbon layer rocking carbon atom. ${ }^{39}$ Specifically, the intensity ratio of $\mathrm{D}$ and $\mathrm{G}$ bands $\left(I_{\mathrm{D}} / I_{\mathrm{G}}\right)$ is indicative of the degree of graphitization in carbon materials (i.e., the lower the value of $I_{\mathrm{D}} / I_{\mathrm{G}}$, the better the microstructure of the char residue is, with fewer defects).$^{\mathbf{4}}$ According to Fig. 13, the $I_{\mathrm{D}} / I_{\mathrm{G}}$ ratios of PS-GO1.0and PS-FGO3.0 are 2.76 and 2.20, respectively. Although GO with lower thermal stability is prone to oxidation, the functionalized flame retardant molecules on FGO surface can catalyze the formation of cohesive char to protect FGO nanosheets from degradation, contributing to the decrease in $I_{\mathrm{D}} / I_{\mathrm{G}}$ ratio. The above SEM and Raman results indicate that both the graphitization degree and thermal stability of the char microstructure are considerably enhanced by the incorporation of FGO. Consequently, the resulting char residue with much improved microstructure can act as effective thermal insulator to retard the release of combustible gases and inhibit the mass and heat transfer.

For a better understanding of the flame retardant action of PS-FGO nanocomposites in condensed phase, FTIR spectroscopy is employed to investigate the char residues of PS-GO1.0 and PS-FGO3.0 (Fig. 14). For PS, peaks at 1382, 753 and $697 \mathrm{~cm}^{-1}$ are assigned to the $\mathrm{C}-\mathrm{H}$ out of plane bending vibration.,39 Concurrently with the intensities of these peaks
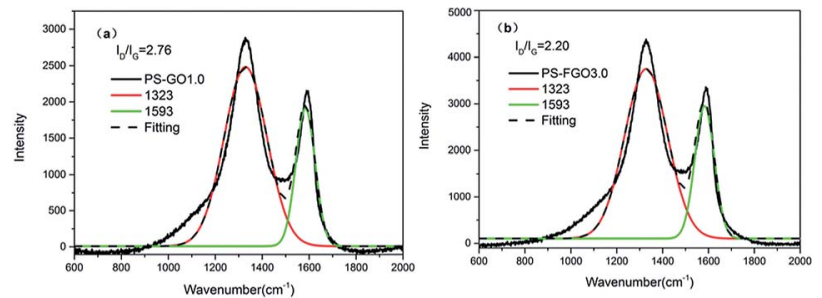

Fig. 13 Raman spectra of char residues for (a) PS-GO1.0 and (b) PSFGO3.0.

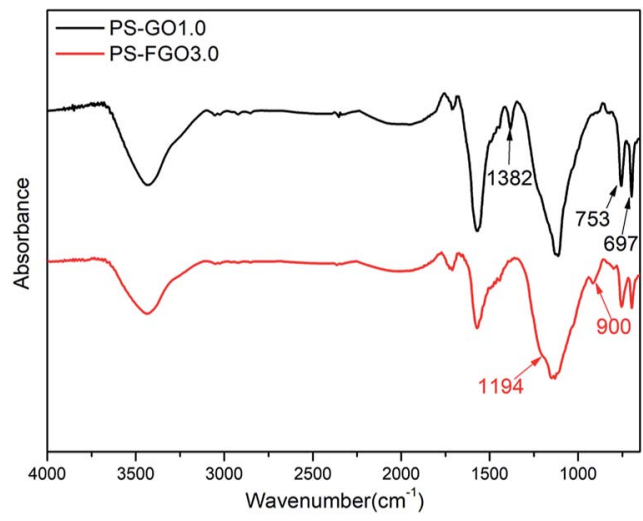

Fig. 14 FTIR spectra of char residues for PS-GO1.0 and PS-FGO3.0.

decrease in PS-FGO3.0, the appearance of new characteristic peaks is observed. Peak at $1194 \mathrm{~cm}^{-1}$, characteristic of $\mathrm{P}-\mathrm{O}-\mathrm{Ar}$, demonstrates the formation of a phosphocarbonaceous structure. ${ }^{40}$ Moreover, another broad peak, ascribed to the asymmetric stretching vibration of $\mathrm{P}-\mathrm{O}-\mathrm{P}$ in polyphosphate species, ${ }^{41}$ can be found at $900 \mathrm{~cm}^{-1}$, indicating that the phosphate species can further undergo cross-linking reaction to form a more complex structure with better thermal stability. Thus, the presence of polyphosphate species is capable of catalyzing the formation of highly structured char, as corroborated by both SEM and Raman spectroscopy. This optimized char residue combined with FGO nanosheets can serve as effective barriers to hinder thermal conductivity, constrain mass transfer and weaken exothermic reaction, improving the flame retardancy of PS nanocomposites in condensed phase.

\section{Conclusions}

In this work, a phosphorus-containing flame retardant (PACP) covalently functionalized graphene oxide was prepared and incorporated into styrene by radical bulk polymerization. Due to good dispersion as well as the characteristic structure of FGO, the resulting PS-FGO nanocomposites demonstrated significant enhancements in thermal properties and flame retardancy. Both the physical hindrance of well dispersed FGO nanosheets and their strong covalent linkages with PS molecular chains contribute a notable $T_{\mathrm{g}}$ increase to PS-FGO nanocomposites as measured by DSC. The TGA results revealed that incorporation of FGO into PS can remarkably promote the char yield at high temperatures and reduce the MMLR, contributing much improved thermal and thermo-oxidative stability. Moreover, much lower values of HRC and THR were seen for samples with FGO from the MCC test, in agreement with an enhancement of the LOI value. Furthermore, TG-FTIR, SEM, Raman spectroscopy and FTIR were employed to gain insight into the flameretardance mechanism of PS-FGO nanocomposites, which explicitly described the different decomposition reactions in PS after incorporation of FGO and corroborated that the presence of FGO in PS can not only decease gaseous volatiles and inhibit the combustion chain reactions in gas phase, but also serve as physical barrier and further react with the polymer molecule 
chains to catalyze the formation of char residue with improved microstructure, exerting flame retardant action in the condensed phase. In short, all the reported results demonstrated the potential of this prepared FGO as an effective flame retardant to enhance the thermal behavior and flame retardancy of PS, providing a foundation for further study.

\section{Conflicts of interest}

There are no conflicts to declare.

\section{Acknowledgements}

This work was financially supported from the Natural Science Foundation of Guangdong Province (Grant No. 2015A030310124).

\section{Notes and references}

1 A. L. Higginbotham, J. R. Lomeda, A. B. Morgan and J. M. Tour, ACS Appl. Mater. Interfaces, 2009, 1, 2256-2261.

2 Y. Yang, C.-E. He, W. Tang, C. P. Tsui, D. Shi, Z. Sun, T. Jiang and X. Xie, J. Mater. Chem. A, 2014, 2, 20038-20047.

3 K. Zhou, W. Yang, G. Tang, B. Wang, S. Jiang, Y. Hu and Z. Gui, RSC Adv., 2013, 3, 25030.

4 S. Stankovich, D. A. Dikin, G. H. Dommett, K. M. Kohlhaas, E. J. Zimney, E. A. Stach, R. D. Piner, S. T. Nguyen and R. S. Ruoff, Nature, 2006, 442, 282-286.

5 Y. Han, T. Wang, X. Gao, T. Li and Q. Zhang, Composites, Part A, 2016, 84, 336-343.

6 D. Zhuo, R. Wang, L. Wu, Y. Guo, L. Ma, Z. Weng and J. Qi, J. Nanomater., 2013, 2013, 1-7.

7 K. Zhou, Z. Gui and Y. Hu, Composites, Part A, 2016, 80, 217227.

8 M. Fang, K. Wang, H. Lu, Y. Yang and S. Nutt, J. Mater. Chem., 2010, 20, 1982.

9 C. Bao, Y. Guo, L. Song and Y. Hu, J. Mater. Chem., 2011, 21, 13942.

10 C. Bao, Y. Guo, B. Yuan, Y. Hu and L. Song, J. Mater. Chem., 2012, 22, 23057.

11 B. Yuan, H. Sheng, X. Mu, L. Song, Q. Tai, Y. Shi, K. M. Liew and Y. Hu, J. Mater. Sci., 2015, 50, 5389-5401.

12 W. Hu, J. Zhan, N. Hong, T. R. Hull, A. A. Stec, L. Song, J. Wang and Y. Hu, Polym. Adv. Technol., 2014, 25, 631-637.

13 Q. Tai, C. Bao, L. Song, S. Nie, Y. Hu and R. K. K. Yuen, Polym. Degrad. Stab., 2010, 95, 830-836.

14 Q. Tai, L. Song, Y. Hu, R. K. K. Yuen, H. Feng and Y. Tao, Mater. Chem. Phys., 2012, 134, 163-169.

15 Q. Tai, L. Song, X. Lv, H. Lu, Y. Hu and R. K. K. Yuen, J. Appl. Polym. Sci., 2012, 123, 770-778.

16 Y. Huafeng, L. Fenghua, S. Changsheng, H. Dongxue, Z. Qixian, N. Li and I. Ari, J. Mater. Chem., 2009, 19, 46324638 .
17 J. H. S. William and R. E. Offeman, J. Am. Chem. Soc., 1958, 80, 1339.

18 Y. Lin, S. Jiang, Z. Gui, G. Li, X. Shi, G. Chen and X. Peng, RSC Adv., 2016, 6, 86632-86639.

19 S. Jiang, Y. Zhu, Y. Hu, G. Chen, X. Shi and X. Qian, Polym. Adv. Technol., 2016, 27, 266-272.

20 W. Hu, B. Yu, S. D. Jiang, L. Song, Y. Hu and B. Wang, J. Hazard. Mater., 2015, 300, 58-66.

21 F. Yinchun, Z. Xiang, X. Zhiqi and W. Yarong, J. Appl. Polym. Sci., 2017, 134, 1-9.

22 M. Fang, K. Wang, H. Lu, Y. Yang and S. Nutt, J. Mater. Chem., 2010, 20, 1982.

23 Y. Zhang, X. Zheng, H. Wang and Q. Du, J. Mater. Chem. A, 2014, 2, 5304.

24 Y. Han, Y. Wu, M. Shen, X. Huang, J. Zhu and X. Zhang, J. Mater. Sci., 2013, 48, 4214-4222.

25 T. G. Fox and P. J. Flory, J. Appl. Phys., 1950, 21, 581-591.

26 B. N. Jang and C. A. Wilkie, The thermal degradation of polystyrene nanocomposite, Polymer, 2005, 46, 2933-2942.

27 Y. Niu, X. Zhang, J. Zhao, Y. Tian, X. Yan and Y. Li, J. Mater. Chem. A, 2014, 2, 10502.

28 K. Dai, L. Song and Y. Hu, High Perform. Polym., 2013, 25, 938-946.

29 X. Wang, L. Song, W. Pornwannchai, Y. Hu and B. Kandola, Composites, Part A, 2013, 53, 88-96.

30 Z. Yang, Z. Gu, X. Yang, Z. Zhang, X. Wang, X. Chen and L. Yang, J. Therm. Anal. Calorim., 2017, 129, 303-314.

31 A. D. La Rosa AR, J. T. Carter and P. T. McGrailBai, Polymer, 1999, 40, 4093-4098.

32 R. E. Lyon, R. N. Walters and S. I. Stoliarov, Polym. Eng. Sci., 2007, 47, 1501-1510.

33 Z. Huiqing, R. J. Farris and P. R. Westmoreland, Macromolecules, 2003, 36, 3944-3954.

34 Y. Guo, C. Bao, L. Song, B. Yuan and Y. Hu, Ind. Eng. Chem. Res., 2011, 50, 7772-7783.

35 B. N. Jang and C. A. Wilkie, Polymer, 2005, 46, 2933-2942.

36 A. Granzow, Acc. Chem. Res., 1977, 11, 177-183.

37 D. Price, L. K. Cunliffe, K. J. Bullett, T. R. Hull, G. J. Milnes, J. R. Ebdon, B. J. Hunt and P. Joseph, Polym. Degrad. Stab., 2007, 92, 1101-1114.

38 D. A. Pethsangave, R. V. Khose, P. H. Wadekar and S. Some, ACS Appl. Mater. Interfaces, 2017, 9, 35319-35324.

39 A. A. Bhutto, D. Vesely and B. J. Gabrys, Polymer, 2003, 44, 6627-6631.

40 B. Serge, L. B. Michel, D. Rene and T. Jean-Michel, J. Chem. Soc., Faraday Trans., 1996, 92, 3435-3444.

41 U. Braun, A. I. Balabanovich, B. Schartel, U. Knoll, J. Artner, M. Ciesielski, M. Döring, R. Perez, J. K. W. Sandler, V. Altstädt, T. Hoffmann and D. Pospiech, Polymer, 2006, 47, 8495-8508. 